\title{
Optical characterization of nonlocal spin transfer torque acting on a single nanomagnet
}

\author{
P. S. Keatley, ${ }^{1, *}$ A. Aziz, ${ }^{2,3}$ M. Ali, ${ }^{4}$ B. J. Hickey,${ }^{4}$ M. G. Blamire, ${ }^{2}$ and R. J. Hicken ${ }^{1}$ \\ ${ }^{1}$ School of Physics and Astronomy, University of Exeter, Stocker Road, Exeter EX4 4QL, United Kingdom \\ ${ }^{2}$ Department of Materials Science and Metallurgy, University of Cambridge, Pembroke Street, Cambridge CB2 3QZ, United Kingdom \\ ${ }^{3}$ Quantum Technology Centre, Department of Physics, University of Lancaster, Lancaster LAI 4YB, United Kingdom \\ ${ }^{4}$ School of Physics and Astronomy, University of Leeds, Leeds LS2 9JT, United Kingdom \\ (Received 11 April 2013; revised manuscript received 5 March 2014; published 21 March 2014)
}

\begin{abstract}
Time-resolved scanning Kerr microscopy (TRSKM) has been used to examine the effect of nonlocal spin transfer torque (NL-STT) within a two-terminal current perpendicular-to-plane nonlocal spin valve. A combination of Oersted field and NL-STT was used to excite and control the magnetization dynamics. By comparison with a macrospin model, the strength of the NL-STT was quantified and found to be comparable to that achieved by direct injection of spin-polarized current. The sensitivity of the magneto-optical probe to small out-of-plane deflections of the magnetization also allows the NL-STT to be quantified in quasi-dc measurements, greatly simplifying the investigation of STT phenomena.
\end{abstract}

DOI: 10.1103/PhysRevB.89.094421 PACS number(s): 85.75.-d, 75.75.-c, 75.78.-n, 78.20.Ls

\section{INTRODUCTION}

Pure spin currents are anticipated to revolutionize electronics. They can transfer information without charge current and so are attractive for energy efficient magnetic devices [1]. A spin current can be generated by the injection of a spin-polarized charge current [2], precessional spin-pumping [3], or the spin Hall [4] and spin Seebeck [5] effects. By spatially separating charge and spin currents within an injection device, a pure spin current can deliver a nonlocal spin transfer torque (NL-STT) to the magnetization of a ferromagnet [6]. Understanding the interaction of a spin current with the magnetization of nanoscale magnetic structures is of fundamental interest and an essential prerequisite for the realization of nonlocal spintronic devices. NL-STT was first detected in magnetoresistance (MR) measurements of the full magnetic reversal of a nanomagnet [7-9]. More recently quantitative MR measurements of STT ferromagnetic resonance have allowed the efficiency of the NL-STT to be characterized [10].

Spintronic devices typically have nanoscale dimensions so as to minimize Oersted field (Oe field) effects and control the spectrum of precessional modes that may be excited [11]. To date, the majority of experimental studies have used MR measurements in three- or four-terminal devices to study these dynamic processes [7-10]. Because of its high spatial $(\sim 300 \mathrm{~nm})$ and temporal ( $\sim 10 \mathrm{ps})$ resolution, time-resolved scanning Kerr microscopy [12] (TRSKM) has proven to be a powerful magneto-optical tool for contactless detection of magnetization dynamics in individual nanomagnets $[13,14]$. Here we use TRSKM to characterize the strength of the NL-STT acting on a NiFe free layer (FL) nanomagnet of a two-terminal current perpendicular-to-plane (CPP) spin valve $[15,16]$. We demonstrate that TRSKM, with carefully optimized spatial resolution and minimal mechanical vibration and drift, can be used as a sensitive, contactless probe of both the dynamic and the quasi-dc magnetization induced by NL-STT. Comparison of the experimental results with a

*p.s.keatley@exeter.ac.uk macrospin model reveals that the strength of the NL-STT is similar to that achieved by injection of spin-polarized charge current.

\section{METHODS}

\section{A. Experimental technique}

In the present study, a microwave coplanar waveguide (CPW) was formed from a $\mathrm{Cu}(200) / \mathrm{Ir}_{20} \mathrm{Mn}_{80}(10) /$ $\mathrm{Co}_{90} \mathrm{Fe}_{10}(12) / \mathrm{Cu}(200) / \mathrm{Ni}_{80} \mathrm{Fe}_{20}(4) / \mathrm{Au}(2)$ spin valve stack by means of photolithography (thickness in $\mathrm{nm}$ ). The CPW was tapered so that the center conductor contained a $20 \mu \mathrm{m}$ long and $2 \mu \mathrm{m}$ wide section. In this narrow section a single CPP pillar was fabricated using three-dimensional focused ion beam (3D-FIB) milling $[15,16]$. The width of the center conductor was first reduced to $\sim 200 \mathrm{~nm}$ by milling from the top. Then, milling from the side was used to define vertical slots through particular layers of the stack, and to remove $\mathrm{NiFe} / \mathrm{Au}$ from either side of the pillar. This method ensures high-quality interfaces and hence efficient spin transfer [8]. During the milling process, the $\mathrm{NiFe} / \mathrm{Au}$ layer was protected by a layer of photoresist, which was later removed to allow for optical access. Previous studies on similar devices have reported no evidence of damage to the ferromagnetic layers due to the ion beam bombardment [16]. A schematic diagram of the device and cross section are shown in Figs. 1(a) and 1(c). In Fig. 1(b) the FIB image shows two dark vertical slots that define a vertical pillar containing an $\mathrm{IrMn} / \mathrm{CoFe}$ reference layer (RL). The two dark horizontal features that extend away from either side of the top of the pillar are the regions in which the NiFe film has been milled away leaving a single NiFe FL nanomagnet directly above the pillar.

Typically TRSKM measurements are performed by passing a pulsed or harmonic current through a CPW so that the associated time-varying Oe field excites magnetization dynamics within an adjoining ferromagnetic sample [12]. In the present work, when a current wave form is passed through the CPW, a charge current passes through the RL and top $\mathrm{Cu}$ contact without passing through the FL. The room-temperature spin diffusion length in $\mathrm{Cu}$ for similar CPP spin valves has been reported to be $\sim 350 \mathrm{~nm}$ [15]. Therefore spin current 


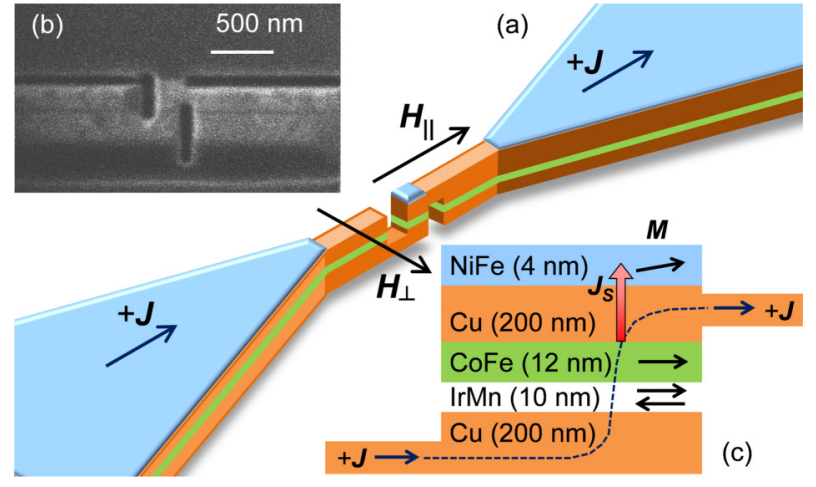

FIG. 1. (Color online) (a) Schematic representation of the CPP pillar showing the parallel $\left(H_{\|}\right)$and perpendicular $\left(H_{\perp}\right)$ geometries. The nonlocal NiFe FL nanomagnet is shown directly above the pillar (blue), while unmilled NiFe remains on the wider parts of the CPW. (b) FIB image of the pillar. (c) Schematic cross section of the pillar in which positive current density is defined.

is expected to diffuse across the $\mathrm{Cu}$ spacer to the $\mathrm{Cu} / \mathrm{NiFe}$ interface, where the resulting spin accumulation is spatially separated from the charge current. Since $\mathrm{NiFe}$ has a low spin resistance the spin current is strongly absorbed by the FL [7], resulting in NL-STT when the FL and RL magnetizations are noncollinear [8]. The current density within the RL is expected to be uniform. The resulting spin current density is also expected to be uniform directly above the RL, but may be slightly reduced for parts of the FL outside of the projected area of the RL. Due to the combination of the thick nonmagnetic spacer layer and the limited optical skin depth, only the FL contributes to the response detected by a focused magneto-optical probe.

TRSKM measurements were performed by synchronizing the pulsed NL-STT and Oe-field excitation (pump) with a probe laser pulse of $400 \mathrm{~nm}$ wavelength. The probe was focused to a diffraction limited spot size of $\sim 300 \mathrm{~nm}$ using a $\times 60$ microscope objective ( 0.85 numerical aperture). Changing the time delay between the pump and probe allowed the out-of-plane component of the dynamic magnetization $\left(m_{\mathrm{z}}\right)$ to be recorded as a function of time by means of the polar Kerr effect. The amplitude of the NL-STT and Oe field were modulated at $\sim 3 \mathrm{kHz}$ so that the polar Kerr signal could be recovered with a lock-in amplifier. Experiments with a quasi-dc NL-STT excitation were performed by replacing the pulsed current wave form with a dc current that was also amplitude modulated from 0 to $I_{\mathrm{dc}}$ at $\sim 3 \mathrm{kHz}$.

Nonlocal devices were fabricated on a sapphire substrate to obtain a current pulse with fast rise time and relaxation within the pillar, and on an $n$-type Si substrate with greater loss and dispersion to obtain a broader current pulse with slower rise time and relaxation. The different substrate allows the time scale over which spin accumulates at the $\mathrm{Cu} / \mathrm{NiFe}$ interface to be modified without the need to change the pulsing electronics used in the experiments. The same CPW dimensions were used for both substrates, and were designed for $\sim 50 \Omega$ characteristic impedance on substrates with dielectric constant of $\sim 10$. The devices fabricated on a $\mathrm{Si} / \mathrm{SiO}_{2}$ substrate had nonlocal nanomagnets with dimensions $390 \times 220 \mathrm{~nm}^{2}$ and $420 \times 200 \mathrm{~nm}^{2}$

(devices A and C) [17] while the device fabricated on a sapphire substrate had dimensions $170 \times 150 \mathrm{~nm}^{2}$ (device B) [18]. A small in-plane bias magnetic field was applied to magnetize the devices either parallel $\left(H_{\|}\right)$or perpendicular $\left(H_{\perp}\right)$ to the length of the CPW, Fig. 1(a).

\section{B. Numerical models \\ 1. Finite-element modeling of charge current and Oersted-field distributions}

The current density within the pillar was estimated to be $2.2 \times 10^{7}$ and $3.3 \times 10^{7} \mathrm{~A} / \mathrm{cm}^{2}$ for devices $\mathrm{A}$ and $\mathrm{B}$, respectively. Finite-element modeling (FEM) was used to understand the charge current distribution within the nonlocal devices. In Fig. 2(a) a vertical cross section along the length of the CPW passing through the center of device B shows the current distribution in the $\mathrm{Cu}$ contacts, the $\mathrm{RL}$, and the FL. The RL is located in the vertical section that is formed by two vertical slots to either side of the pillar. As shown in Fig. 2(a), the position of the RL ensures that the charge current is almost perpendicular to the RL with quite uniform density of $\sim 3.3 \times 10^{7} \mathrm{~A} / \mathrm{cm}^{2}$.

The Oe field associated with the charge current was also calculated using the FEM current distribution. Figure 2(b) shows the in-plane component of the Oe field that lies perpendicular to the CPW. The distribution is shown for three horizontal planes. The top and bottom planes are coplanar with the top and bottom surfaces of the $\mathrm{Cu}$ contacts, respectively. They reveal that the in-plane Oe field is perpendicular to the CPW and approximately uniform along its length, The

(a)

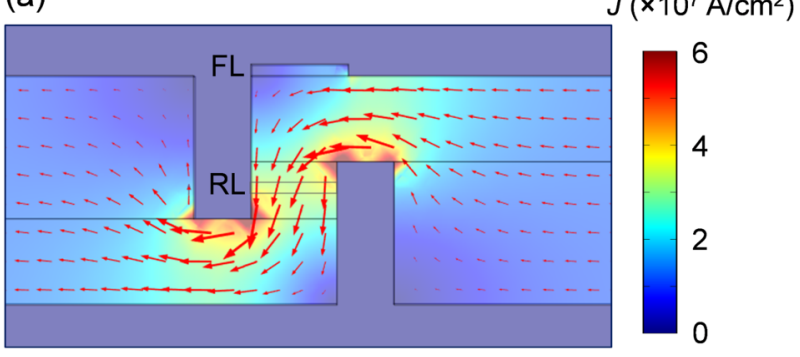

(b)

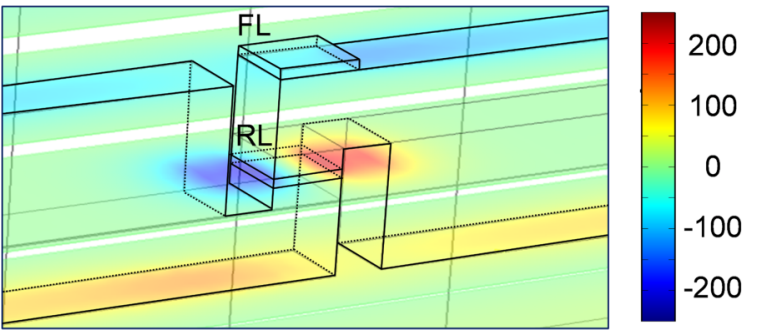

FIG. 2. (Color online) (a) Finite-element model of the charge current distribution in device B. (b) In-plane component of the Oe field perpendicular to the $\mathrm{CPW}$ calculated from the current distribution in (a) and shown in three horizontal planes. The planes are positioned at the top surface of the $\mathrm{Cu}$ contacts passing through the FL, at the center of the pillar passing through the RL, and at the bottom surface of the $\mathrm{Cu}$ contacts. 
amplitude of the Oe field was estimated to be $\sim 46$ and $\sim 70$ Oe for devices A and B, respectively. The Oe field exerts maximum (minimum) initial torque when the nanomagnet is magnetized parallel (perpendicular) to the CPW. Consequently the perpendicular geometry is preferred for the detection of the NL-STT.

The third plane passes through the center of the vertical pillar and shows the in-plane Oe field acting on the RL. As expected for a conductor with uniform current density, the Oe field is found to vary linearly across the RL with maximum strength at the edges of the RL. However, the Oe field curls around the current and so the direction of the Oe field is opposite on opposite sides of the RL. A similar but orthogonal distribution is found for the component of the Oe field parallel to the length of the CPW. In fact, the CPP nonlocal geometry simplifies the spatial character of the Oe field acting on the FL making it more amenable to macrospin simulations used later in this work. In contrast, the FL in current injection devices is subject to an Oe field that curls around the current direction exerting highly nonuniform torque on the magnetization.

\section{Micromagnetic modeling of the Oe-field excitation of the reference layer magnetization}

To understand the effect of the circulating Oe field on the magnetization within the RL, micromagnetic simulations were performed using the object-oriented micromagnetic framework (OOMMF) [19]. A $220 \times 220 \times 12 \mathrm{~nm}^{3}$ nanomagnet, with rounded corners of $30 \mathrm{~nm}$ radius, was simulated using a cell size of $5 \times 5 \times 12 \mathrm{~nm}^{3}$ and exchange parameter of $3 \times 10^{-7} \mathrm{ergs} / \mathrm{cm}$. The saturation magnetization, $g$ factor, and Gilbert damping parameter $\alpha$ were assumed to have values of $1800 \mathrm{emu} / \mathrm{cm}^{3}, 2.1$, and 0.01 , respectively. The RL magnetization was excited using a spatially circulating pulsed magnetic field with 30 ps rise time and 70 ps duration, Fig. 3(a). The peak amplitude varied linearly from 0 Oe at the center of the RL to 200 Oe at the edges as in the results of the FEM. The spatial variation of the Oe field, shown in Figs. 3(b) and 3(c) for the $x$ and $y$ component, respectively, was defined by the expression for a magnetic field within a cylindrical current-carrying conductor with uniform current density.

Due to the square shape of the RL, the equilibrium magnetization configuration formed an $S$ state where the magnetization is canted at both edges of the element that lie perpendicular to the applied magnetic field, Fig. 3(d). The transitions between these canted regions and the central region are pinned by the top-left and lower-right corners of the square element (even though rounded corners are included in the model) preventing the opposite ends of the RL from switching in response to the pulsed and circulating Oe field. The maximum perturbation to the magnetization is shown in Fig. 3(e), which demonstrates that the RL retains its quasi-uniform single-domain equilibrium state in spite of the highly nonuniform Oe field. This is in contrast to the case of a circular element (not shown) in which a vortex equilibrium state can form easily since the transitions between the central region and the canted end regions are not pinned by the shape of the element. Following excitation of the square RL element, the magnetization subsequently relaxes to the equilibrium state in Fig. 3(a). Therefore, the macrospin model used later in this
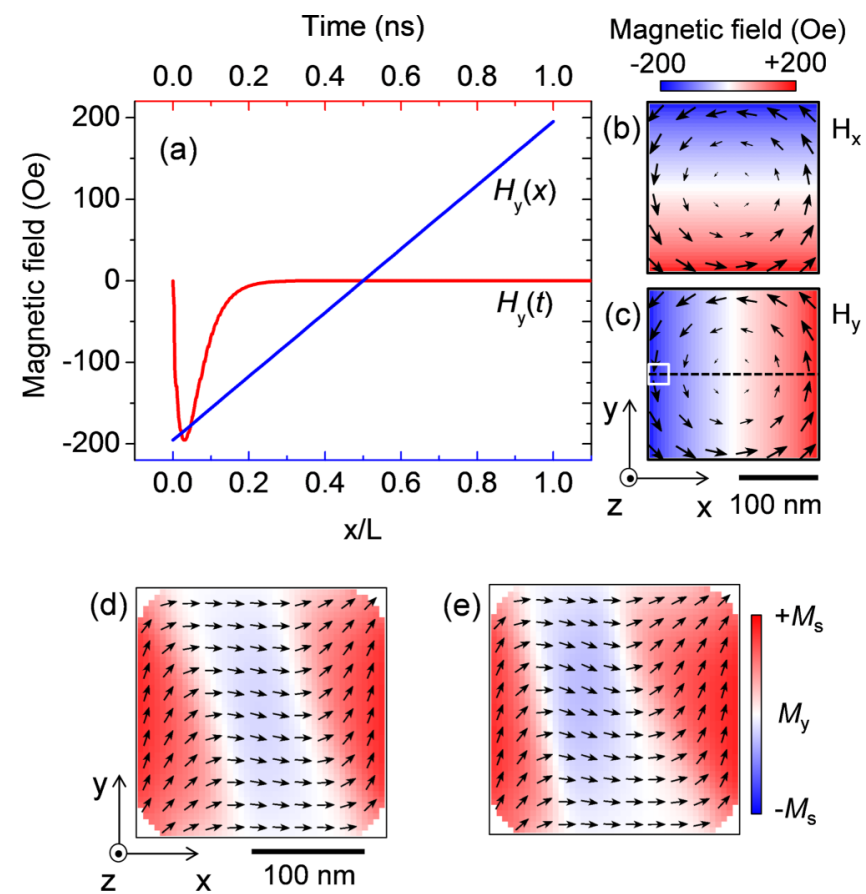

(e)

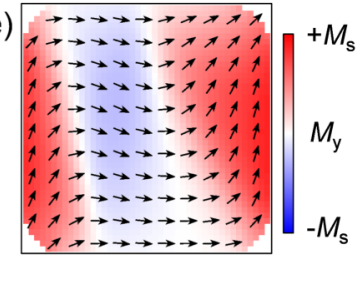

FIG. 3. (Color online) (a) Spatial and temporal profiles of the spatially circulating pulsed Oe field. (b) and (c) Spatial variation of the $x$ and $y$ components of the field, respectively. The spatial and temporal profiles in (a) are extracted from the dashed line and white box in (c), respectively. (d) Equilibrium $S$-state magnetization configuration of the RL. (e) Maximum perturbation of the magnetization in response to a pulsed circulating Oe field. The red-white-blue color map represents $-M_{\mathrm{s}}, 0,+M_{\mathrm{s}}$ for the $y$ component of the magnetization.

work is a reasonable approximation for the case of a square RL element.

\section{Macrospin simulations}

Macrospin simulations [20] have been performed in order to understand the dynamic response of the FL to the combined STT and Oe-field excitation. Strictly speaking the nonlocal nanopillar should be treated micromagnetically. However, micromagnetic simulations show that edge modes occupy the majority of the FL volume when the bias field is small, so that the assumption of a quasi-uniform response is a reasonable approximation. Macrospin simulations allow a simple examination of torque exerted on the FL and therefore an estimate of the spin torque efficiency. The RL was also subject to STT, but since the Oe field has an azimuthal spatial variation it was assumed to have zero average value in the macrospin model. The FL equation of motion had the form

$$
\begin{aligned}
-\frac{1}{\left|\gamma_{f}\right|} \frac{\partial \mathbf{M}_{f}}{\partial t}= & \mathbf{M}_{f} \times \mathbf{H}_{\mathrm{eff}}+\frac{\alpha_{f}}{M_{f}} \mathbf{M}_{f} \times \frac{\partial \mathbf{M}_{f}}{\partial t} \\
& -\frac{J \hbar P \eta}{2|e| d_{f} M_{f}^{2}} \mathbf{M}_{f} \times\left(\mathbf{M}_{f} \times \hat{\mathbf{M}}_{r}\right),
\end{aligned}
$$

where $\gamma_{f}$ is the FL gyromagnetic ratio, $\mathbf{M}_{f, r}$ is the magnetization of the FL $(f)$ and RL $(r)$ macrospins, $\alpha_{f}$ is the Gilbert damping parameter for the FL, $J$ and $P$ are the density and spin polarization of the current respectively, $\eta$ describes 
the efficiency of the spin transfer, $e$ is the electron charge, and $d_{f}$ is the thickness of the FL. $\mathbf{H}_{\text {eff }}$ is the total effective magnetic field that includes contributions from the external, pulsed, and anisotropy fields. A similar equation of motion with the $f$ and $r$ subscripts interchanged describes the motion of the RL magnetization. The RL was subject to a weak exchange bias field of $10 \mathrm{Oe}$. This is reasonable since ion bombardment during the milling process is known to lead to reduced exchange bias fields [16,21]. The dipolar coupling field between the FL and RL was assumed to be negligible. From previous studies of similar devices, the magnetization and uniaxial anisotropy field were assumed to be $860 \mathrm{emu}$ $\mathrm{cm}^{-3}$ and $5 \mathrm{Oe}$ for the FL, and $1800 \mathrm{emu} \mathrm{cm}^{-3}$ and $167 \mathrm{Oe}$ for the RL, respectively [22]. For both layers the values of $\alpha$, the $g$ factor, and $P$ were assumed to be 0.03 [13], 2, and 0.45 [23], respectively, while $\eta$ was varied to achieve the best agreement with the experiment. The value of $\eta$ for the RL was found to have negligible effect upon the FL dynamics and was fixed at 0.1 [10]. The experimental values for the maximum current density were assumed, while the value of the in-plane Oe field was extracted from the FEM. The transmitted current pulse [Figs. 5(a) and 5(b)] was used to define their temporal profile.

\section{RESULTS AND DISCUSSION}

\section{A. Time-resolved Kerr measurements}

Figures 4(a) and 4(b), respectively, show TR reflectivity and TR Kerr images of device B for three time delays indicated

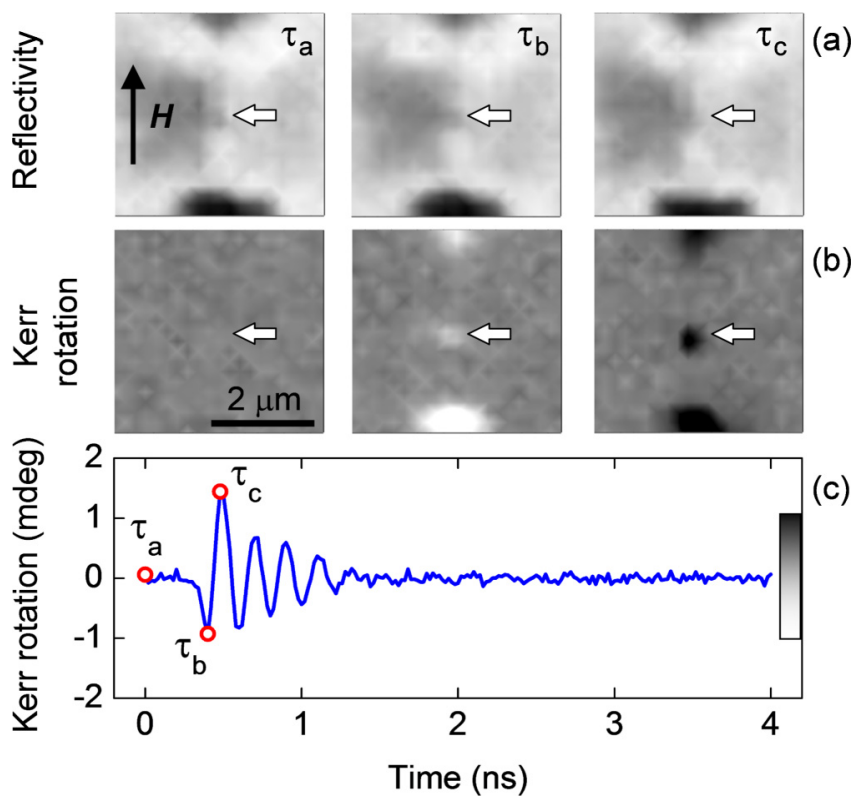

FIG. 4. (Color online) TR scanning images of reflectivity (a) and Kerr rotation (b) for the $170 \times 150 \mathrm{~nm}^{2}$ nanomagnet of device $\mathrm{B}$. The position of the nonlocal FL nanomagnet is indicated by the white arrow. Images were acquired before the arrival of the pump at $\tau_{\mathrm{a}}$, and at the first $\left(\tau_{\mathrm{b}}\right)$ and second $\left(\tau_{\mathrm{c}}\right)$ antinodes of precession, as shown in (c) by the open red symbols labeling the TR Kerr signal acquired from the nanomagnet. The gray scale for the Kerr images is shown in (c). The data were acquired in the parallel geometry at a field value of 200 Oe for positive current direction $+J$.
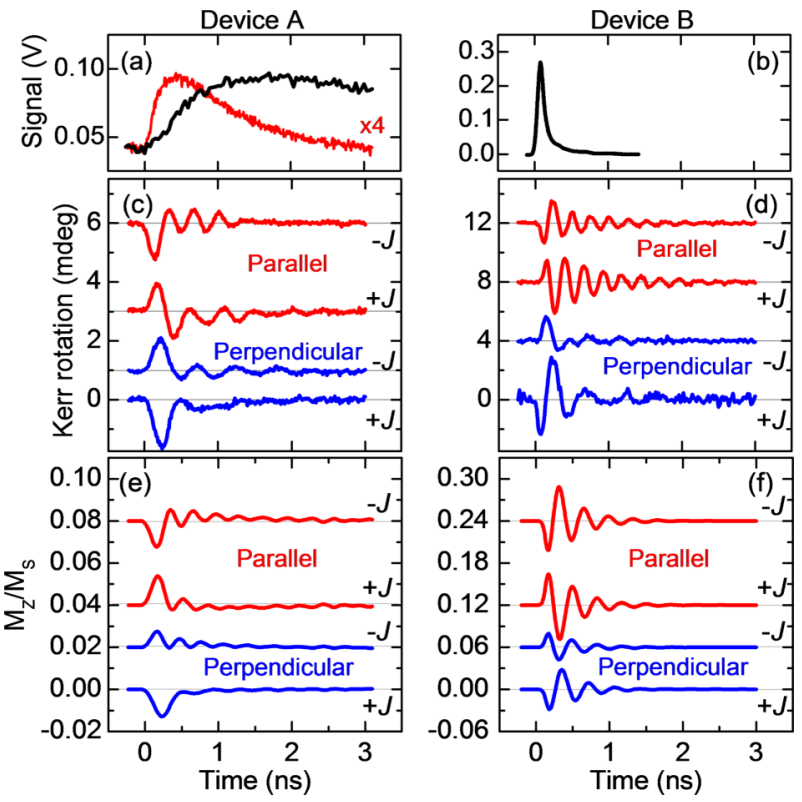

FIG. 5. (Color online) Transmitted current pulse for device A (a) and device B (b) (black curves). For device A, a faster temporal profile $(\times 4$, red curve) used in macrospin simulations is also shown in (a). TR Kerr signals acquired from device A (c) and B (d) are shown for the parallel (red traces) and perpendicular (blue traces) geometries and for opposite directions of the pulsed current $( \pm J)$. In (e) and (f) TR traces generated using a macrospin model are shown. In (c) to (f) the bias field strength was 100 Oe.

on the TR signal shown in Fig. 4(c). At time delay $\tau_{\mathrm{a}}$ the optical probe pulse arrives before the NL-STT and Oe-field pump and so no polar Kerr contrast is observed. Following the arrival of the pump, magnetization dynamics within the FL nanomagnet are clearly observed in Kerr images of opposite contrast acquired at the first $\tau_{\mathrm{b}}$ and second $\tau_{\mathrm{c}}$ antinodes of the precession signal shown in Fig. 4(c). The FL nanomagnet is clearly resolved from the unmilled $\mathrm{NiFe}$ film that gives rise to strong contrast at top and bottom of images for $\tau_{\mathrm{b}}$ and $\tau_{\mathrm{c}}$. The static and dynamic dipolar interaction between the FL nanomagnet and unmilled $\mathrm{NiFe}$ is expected to be negligible from the results of micromagnetic simulations.

The transmitted current pulse recorded by a $50 \mathrm{GHz}$ sampling oscilloscope is shown for device A and B in Figs. 5(a) and 5(b), respectively. The attenuation and broadening of the pulse in device A results from loss in the $n$-type Si substrate. In device $\mathrm{B}$ a much shorter pulse with reduced attenuation was observed. The incident pulse amplitude was $5.2 \mathrm{~V}$ with a duration of 80 ps for device $\mathrm{A}$, while for device $\mathrm{B}$ the incident amplitude was attenuated to $560 \mathrm{mV}$ to achieve similar peak current density within each device. In device A the combined effects of the NL-STT and Oe field are present for the duration of the TR scan, while in device B they are present for the first 200-300 ps only.

The TR signals acquired from devices A and B are shown in Figs. 5(c) and 5(d), respectively, for both the parallel and perpendicular geometries, and for both directions of the pulsed current $\pm J$. A bias field strength of 100 Oe was used in all cases to ensure a detectable precession signal and avoid the coexistence of center and edge modes within the FL 
nanomagnet [24]. Figures 5(c) and 5(d) show that the initial torque has opposite sign for opposite current directions $\pm J$ as expected when a significant Oe field is present. This suggests that the equilibrium magnetizations of the FL and the RL are canted relative to the bias field, otherwise no Oe-field torque is expected in the perpendicular geometry. Canting of the magnetization is typical of confined magnetic structures in small bias fields [24].

In the parallel geometry, TR signals obtained from device A [Fig. 5(c)] have similar relaxation times, but frequencies of 2.1 and $2.7 \mathrm{GHz}$ for $+J$ and $-J$ respectively. For device B [Fig. 5(d)] the same frequency of $3.9 \mathrm{GHz}$ is observed for $\pm J$. The different frequencies for devices $\mathrm{A}$ and $\mathrm{B}$ may be attributed to the different sizes of the FL nanomagnet, where frequency increases as size decreases due to the increased in-plane demagnetizing field [24,25]. The change in frequency for device $\mathrm{A}$ for $\pm J$ may result from the longer relaxation time of the Oe-field contribution. Since the magnetization is canted with respect to the bias field, the addition of the Oe field leads to total effective fields of different magnitude acting upon the magnetization for $\pm J$ as confirmed by micromagnetic simulations. While a field-like STT could influence the frequency, this is expected to be negligible in a metallic spin valve.

In the perpendicular geometry, the amplitude of precession following the initial, larger amplitude, half cycle is smaller than in the parallel geometry. However, the reduced Oe-field torque allows the effect of the NL-STT to be more clearly seen. In device A, the precession is more heavily (weakly) damped for $+J(-J)$ as expected when in-plane STT modifies the damping [26]. This effect is not clearly observed in device $\mathrm{B}$, for which the NL-STT is short lived.

Figures 5(e) and 5(f) show the TR traces generated by the macrospin simulations corresponding to the experimental signals of Figs. 5(c) and 5(d). The canting of the equilibrium state magnetization was estimated from micromagnetic simulations and included in the macrospin simulations by rotating the Oe field with respect to the static magnetization. The misalignment between the static magnetization and the bias field for devices $\mathrm{A}$ and $\mathrm{B}$ was $10^{\circ}$ and $9^{\circ}$ in the parallel geometry and $30^{\circ}$ and $25^{\circ}$ in the perpendicular geometry respectively. The canting is in fact necessary to reproduce the initial, larger amplitude, half cycle of precession observed in the experimental signals for both devices. For device A it was also necessary to compress the temporal profile of the pulse $\times 4$ [Fig. 5(a)] to reproduce the shape of the signal during the first cycle. It is reasonable to assume that the rise of the current pulse at the device location at the center of the waveguide will be faster than that of the transmitted pulse since broadening occurs between the device and the oscilloscope. The simulations show substantial correlation with the experimental signals, in spite of small quantitative differences in the amplitude, frequency, and relaxation that are expected since the simulations assume a single macrospin.

The STT term can be switched off in the macrospin simulations by setting $P=0$, allowing the effects of STT and the Oe field to be distinguished. For devices A and B in the parallel geometry, the macrospin simulations show no change in frequency when the STT is turned off, as expected in the absence of a field-like STT. However, for Device A, the
STT was found to slightly increase (decrease) the amplitude of the TR traces for $-J(+J)$ in agreement with the small difference in amplitude of the experimental TR signals. As expected, the effect of the NL-STT on the dynamic response of the FL is most clearly observed in the perpendicular geometry, in which the Oe field contribution is minimized. For device $\mathrm{B}$ the simulations revealed very little difference in amplitude for $\pm J$ although a more significant difference was observed experimentally. Since the NL-STT is only present for $\sim 200 \mathrm{ps}$ in device B, the STT is not expected to have a significant effect on the amplitude at larger time delay. The difference in amplitude in the experiment may be attributed to a more complicated micromagnetic equilibrium state.

In addition to the changes in frequency, amplitude, and damping, the TR traces from device A also show a vertical offset from zero following the initial half cycle. Examination of the torques generated in the macrospin simulation reveals that the offset is due to out-of-plane canting of the magnetization resulting from an out-of-plane torque when the STT is active. The in-plane STT rotates the magnetization in plane to a nonequilibrium configuration with respect to the bias and anisotropy fields thus yielding an out-of-plane torque. For device A [Fig. 5(c)], the precession signal is offset for both the parallel and perpendicular geometries. However, for device B [Fig. 5(d)] no offset is present since the NL-STT is short lived. Similarly, in Figs. 5(e) and 5(f) the simulations reveal an offset for device A, but not for device B. While an offset of the simulated precession signal is observed in device $A$ when the STT is switched off $(P=0)$, the sign and amplitude of the offset do not agree with experiment. When STT is present $(P=0.45)$, the sign and amplitude of the offset can be matched to the experiment by adjusting $\eta$, where only quantitative agreement of the vertical offset is necessary to estimate the value of $\eta$.

In Fig. 6(a) the simulated TR traces for device A in the perpendicular geometry are overlaid with the experimental signal. Simulated traces are shown for $P=\eta=0$ (STT off), and for $P=0.45$, and $\eta=1,0.5,0.4$, and 0.3 . The value of $\eta$, or strength of the STT, was found to affect the offset of the precession following the initial large-amplitude half cycle and was determined by adjusting $\eta$ so that the relative height of the initial half cycle and the transient offset were in agreement. The sum of the squared residuals for TR signals simulated for $0<\eta$ $<1$ was found to be minimized for the value of $\eta=0.4 \pm 0.1$. This value is $\times 4$ larger than a value recently reported for a nonlocal geometry [10], but similar to those achieved by direct injection of spin-polarized current in a point contact geometry [23]. The simulated traces in Figs. 5(e) and 5(f) all assume $P=0.45$ and $\eta=0.4$, for which the offset of the experimental precession signals in Figs. 5(c) and 5(d) are well reproduced.

\section{B. Quasi-static Kerr measurements}

To experimentally isolate the NL-STT from the Oe field, quasi-dc experiments were performed using a current density of $4.2 \times 10^{7} \mathrm{~A} / \mathrm{m}$. Since the rise time of the modulated current is slow and the frequency low, the Oe field adds to the total effective field and rotates the magnetization in-plane only, thus eliminating its contribution to the polar Kerr signal. The STT then rotates the magnetization in-plane away from the 

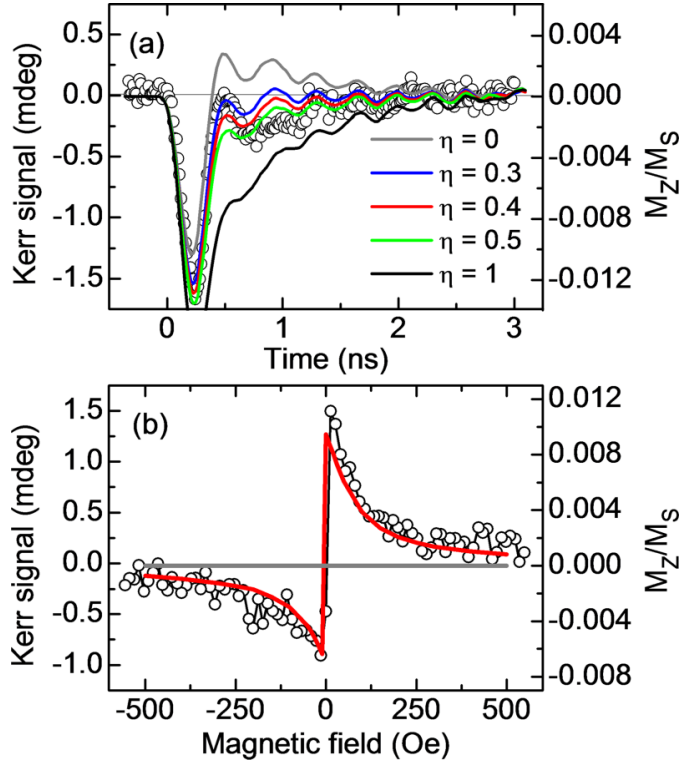

FIG. 6. (Color online) (a) Experimental TR signal (open symbols) acquired from device $\mathrm{A}$ in the perpendicular geometry for $+J$ showing significant damping of the dynamic response to a pulsed NL-STT and Oe-field excitation. (b) Quasi-dc response to the NL-STT as a function of bias field. In (a) and (b) simulated traces are shown as solid curves.

effective field yielding an out-of-plane torque. The resulting out-of-plane component of the magnetization leads to a polar Kerr signal that depends upon the NL-STT only. In Fig. 6(b) the polar Kerr signal acquired from device $\mathrm{C}$ is shown as a function of the in-plane magnetic field in the parallel geometry. The field is swept from 500 Oe where the STT is weak due to strong parallel alignment of the FL and RL. As the field is reduced the FL and RL become misaligned, increasing the strength of the NL-STT and hence the polar Kerr signal. The out-of-plane signal is larger than the offset observed in Fig. 6(a) owing to the larger current density used. Around zero field both the FL and RL switch. Macrospin simulations assuming $P=0.45$ and $\eta=0.4$ are in excellent agreement with the form of the experimental signal, confirming the contactless detection of $\mathrm{dc}$ NL-STT. When $P=\eta=0$, no effect is observed (grey curve), confirming that the response is due to NL-STT rather than the Oe field.

\section{SUMMARY}

In summary, two-terminal CPP spin valves with a thick spacer layer have been fabricated by 3D-FIB milling and used to observe NL-STT. TRSKM was used in a pulsed and quasi-dc mode of operation for contactless detection of NL-STT through its effect on the FL magnetization. Macrospin simulations have been used to estimate the strength of the NL-STT, which is larger than previously reported for a nonlocal geometry, but of similar magnitude to that achieved by direct injection of spin-polarized current. The contactless characterization of NL-STT is an important experimental development that reduces the complexity of device fabrication required to study STT. In the absence of the Oe-field contribution, the quasi-dc detection may allow the predicted angular dependence of $\eta$ [27] to be characterized for local and nonlocal spin valves that are expected to exhibit different angular dependence of the STT [28,29]. Furthermore quasi-dc detection of STT can be performed with continuous-wave lasers, making the study of NL-STT accessible without the need for high-frequency measurement apparatus.

\section{ACKNOWLEDGMENTS}

The authors gratefully acknowledge the financial support from the Engineering and Physical Sciences Research Council through the Spin@RT Consortium Grant No. EP/D000572/1 and Grant No. EP/I038470/1, and from the EU Grant No. MASTER NMP-FP7-212257.
[1] T. Kimura, N. Hashimoto, S. Yamada, M. Miyao, and K. Hamaya, NPG Asia Mater. 4, e9 (2012).

[2] M. Johnson and R. H. Silsbee, Phys. Rev. Lett. 55, 1790 (1985).

[3] Y. Tserkovnyak, A. Brataas, and G. E. W. Bauer, Phys. Rev. Lett. 88, 117601 (2002).

[4] Y. K. Kato, R. C. Myers, A. C. Gossard, and D. D. Awschalom, Science 306, 1910 (2004).

[5] K. Uchida, J. Xiao, H. Adachi, J. Ohe, S. Takahashi, J. Ieda, T. Ota, Y. Kajiwara, H. Umezawa, H. Kawai, G. E. W. Bauer, S. Maekawa, and E. Saitoh, Nat. Mater. 9, 894 (2010).

[6] Y. Otani and T. Kimura, Philos. Trans. R. Soc. London Ser. A 369, 3136 (2011).

[7] T. Kimura, Y. Otani, and J. Hamrle, Phys. Rev. Lett. 96, 037201 (2006).

[8] T. Yang, T. Kimura, and Y. Otani, Nat. Phys. 4, 851 (2008).

[9] J. Z. Sun, M. C. Gaidis, E. J. O’Sullivan, E. A. Joseph, G. Hu, D. W. Abraham, J. J. Nowak, P. L. Trouilloud, Y. Lu, S. L.
Brown, D. C. Worledge, and W. J. Gallagher, Appl. Phys. Lett. 95, 083506 (2009)

[10] L. Xue, C. Wang, Y.-T. Cui, L. Liu, A. Swander, J. Z. Sun, R. A. Buhrman, and D. C. Ralph, Phys. Rev. Lett. 108, 147201 (2012).

[11] V. E. Demidov, S. Urazhdin, H. Ulrichs, V. Tiberkevich, A. Slavin, D. Baither, G. Schmitz, and S. O. Demokritov, Nat. Mater. 11, 1028 (2012).

[12] P. S. Keatley, P. Gangmei, M. Dvornik, R. J. Hicken, J. Grollier, C. Ulysse, J. R. Childress, and J. A. Katine, Top. Appl. Phys. 125, 17 (2013).

[13] P. S. Keatley, P. Gangmei, M. Dvornik, R. J. Hicken, J. R. Childress, and J. A. Katine, Appl. Phys. Lett. 98, 082506 (2011).

[14] P. S. Keatley, P. Gangmei, M. Dvornik, R. J. Hicken, J. Grollier, and C. Ulysse, Phys. Rev. Lett. 110, 187202 (2013).

[15] C. W. Leung, C. Bell, G. Burnell, and M. G. Blamire, Phys. Rev. B 72, 212409 (2005). 
[16] M. C. Wu, A. Aziz, J. D. S. Witt, M. C. Hickey, M. Ali, C. H. Marrows, B. J. Hickey, and M. G. Blamire, Nanotechnology 19, 485305 (2008).

[17] Due to the longevity of the devices, two devices of similar dimensions were studied on the $\mathrm{Si} / \mathrm{SiO} 2$ substrate.

[18] TR signals and images acquired from the smaller device B demonstrate the spatial resolution and mechanical stability of the TRSKM.

[19] M. Donahue and D. G. Porter, OOMMF User's Guide, Version 1.0, NISTIR 6376 (National Institute of Standards and Technology, Gaithersburg, MD, 1999), see http://math.nist.gov/oommf

[20] M. D. Stiles and J. Miltat, in Spin Dynamics in Confined Magnetic Structures III, edited by B. Hillebrands and A. Thiaville, Vol. 101 (Springer-Verlag, Berlin, 2006), p. 225.

[21] M. C. Wu, A. Aziz, D. Morecroft, M. G. Blamire, M. C. Hickey, M. Ali, G. Burnell, and B. J. Hickey, Appl. Phys. Lett. 92, 142501 (2008).
[22] N. Banerjee, A. Aziz, M. Ali, J. W. A. Robinson, B. J. Hickey, and M. G. Blamire, Phys. Rev. B 82, 224402 (2010).

[23] M. R. Pufall, W. H. Rippard, and T. J. Silva, Appl. Phys. Lett. 83, 323 (2003).

[24] P. S. Keatley, V. V. Kruglyak, A. Neudert, E. A. Galaktionov, R. J. Hicken, J. R. Childress, and J. A. Katine, Phys. Rev. B 78, 214412 (2008).

[25] V. V. Kruglyak, A. Barman, R. J. Hicken, J. R. Childress, and J. A. Katine, Phys. Rev. B 71, 220409(R) (2005).

[26] Z. Li and S. Zhang, Phys. Rev. B 68, 024404 (2003).

[27] J. C. Slonczewski, J. Magn. Magn. Mater. 159, L1 (1996).

[28] T. Yang, A. Hirohata, T. Kimura, and Y. Otani, Phys. Rev. B 74, 153301 (2006).

[29] G. E. W. Bauer, Y. Tserkovnyak, D. Huertas-Hernando, and A. Brataas, Phys. Rev. B 67, 094421 (2003). 